\title{
Research on the propagation of partially coherent cosh-Gaussian beams through an $A B C D$ optical system in non-Kolmogorov turbulence by effective tensor approach
}

\author{
HongJie MA, Jinsong LI*, YueyANG CHEN \\ College of Optical and Electronic Technology China Jiliang University, \\ Hangzhou 310018, China
}

${ }^{*}$ Corresponding author: Jinsong Li, lijinsong@cjlu.edu.cn

\begin{abstract}
An efficient tensor approach is used to study the propagation of partially coherent cosh-Gaussian beams through an $A B C D$ optical system in non-Kolmogorov turbulence. Analytical expressions for the average intensity of the beam propagation are derived. The properties of the average intensity are investigated with a numerical example. One finds that the propagation of the beam with larger spatial coherence length is less affected by distance when the propagation distance is long enough, and as the Ch-parameter increases, the beam propagation is less effected by turbulent atmosphere. It is also found that the average intensity distribution of the cosh-Gaussian beams with larger spatial correlation length is more affected by the structure constant of turbulence (i.e., turbulence level). By choosing a suitable Ch-parameter and spatial coherence length, the partially coherent cosh-Gaussian beams can be better transmitted in non-Kolmogorov turbulence. Our results will be useful in free-space communication.
\end{abstract}

Keywords: partially coherent cosh-Gaussian beam, $A B C D$ optical system, non-Kolmogorov turbulence.

\section{Introduction}

The propagation characteristics of a variety of laser beams in turbulent atmosphere have been studied extensively due to their wide applications, such as long-distance optical communications, imaging, remote sensing, etc. [1-ㅁ] . Kolmogorov's power spectrum of refractive index fluctuations is widely accepted and employed in previous studies. However, experimental results reveal that turbulence in portions of the troposphere and the stratosphere deviates greatly from Kolmogorov's model [ $\underline{5}-\underline{7}]$. Therefore, it is very important to find other models more general than the Kolmogorov model 
to describe non-Kolmogorov turbulence. Then a non-Kolmogorov model is presented in $[\underline{8}, \underline{9}]$, which reduces to the Kolmogorov model only for the power-law exponent value. Nevertheless, based on this model, a great deal of work has been investigated: the scintillation index, the signal-to-noise ratio, and the bit-error rate [10], the average spreading of a Gaussian-Schell model beam [11], the second-order statistics of stochastic electromagnetic beams [12]. On the other hand, in some practical systems, such as laser radar systems and remote sensing systems, etc., paraxial $A B C D$ optical systems such as thin lenses are commonly encountered [ $\underline{13}-\underline{15}]$. Therefore, it is very important to study the propagation of laser beams through an $A B C D$ optical system in turbulent atmosphere.

CASPERSON and TOVAR demonstrated that the Hermite-sinusoidal-Gaussian (HSG) beam is a set of solutions for the wave equation in Cartesian coordinates system [16] and derives its propagation formula. As one of the special cases of HSG beams, the hyperbolic-cosine-Gaussian beam (ChGB) has been studied in recent years due to its potential applications in remote sensing, imaging and communication [17-19]. As far as we know, no results have been reported up until now on propagation of a partially coherent ChGB through a paraxial $A B C D$ optical system in non-Kolmogorov turbulence. And more importantly, we are the first to use an efficient tensor approach (ETA) [르, 21] to simulate the propagation of partially coherent ChGB and illustrate it by a numerical example, because the ETA has obvious advantages over traditional methods. In the traditional discrete Fourier transform (DFT) related methods, since the integral window cannot be changed during the two Fourier transforms, the loss of high frequency information after the quadratic transformation may cause distortions in the reconstruction by DFT [22]. Furthermore, sampling constraints must be strictly obeyed when taking the DFT [23]. Any violation of the constraints would result in errors in reconstruction. ETA has improved both. One is to replace the integral operation with matrix multiplication, and there is no need to consider the influence of the integration window. The second is to use the sampling of points replaced by small -segment integral sampling. Under the same sample size, the calculation time of ETA is greatly shortened and the calculation result is more accurate.

In this paper, the dependences of the average intensity distribution on the spatial correlation length $\sigma_{0}$, the Ch-parameter $\Omega_{0}$, the propagation distance $z$ and the structure constant of turbulence $C_{n}^{2}$ are mainly discussed.

\section{Theory}

In the Cartesian coordinate system, the $z$-axis is taken to be the propagation axis. The cross-spectral density (CSD) of partially coherent ChGB in the source plane $z=0$ takes the form as [24]

$$
W\left(x_{1}, x_{2}, y_{1}, y_{2}, 0\right)=W\left(x_{1}, x_{2}, 0\right) W\left(y_{1}, y_{2}, 0\right)
$$

with $W\left(x_{1}, x_{2}, 0\right)$ and $W\left(y_{1}, y_{2}, 0\right)$ given by 


$$
W_{0}\left(i_{1}, i_{2}, 0\right)=\exp \left(-\frac{i_{1}^{2}+i_{2}^{2}}{w_{0}^{2}}\right) \cosh \left(\Omega_{0} i_{1}\right) \cosh \left(\Omega_{0} i_{2}\right) \exp \left(-\frac{\left(i_{1}-i_{2}\right)^{2}}{2 \sigma_{0}^{2}}\right)
$$

where $j=x$ or $y, w_{0}$ denotes the waist width of the Gaussian part, $\Omega_{0}$ is the parameter associated with the Ch-part, $\sigma_{0}$ is the spatial correlation length of the laser source in the plane $z=0$.

Based on Huygens-Fresnel diffraction integral, the cross-spectral density transmission of partially coherent ChGB passing through an $A B C D$ optical system in turbulent atmosphere can be expressed as [20]

$$
\begin{aligned}
\langle I(x, y, z)\rangle & =W(x, x, y, y, z) \\
=\left(\frac{k}{2 \pi B}\right)^{2} & \int_{-\infty}^{+\infty} \int_{-\infty}^{+\infty} \int_{-\infty}^{+\infty} \int_{-\infty}^{+\infty} W_{0}\left(x_{1}, x_{2}, y_{1}, y_{2}, 0\right) \\
& \times \exp \left\{-\frac{i k}{2 B}\left[A\left(x_{1}^{2}-x_{2}^{2}+y_{1}^{2}-y_{2}^{2}\right)-2 x\left(x_{1}-x_{2}\right)-2 y\left(y_{1}-y_{2}\right)+D x^{2}\right]\right\} \\
& \times\left\langle\exp \left[\psi\left(x_{1}, y_{1}, x, y, z\right)+\psi^{*}\left(x_{2}, y_{2}, x, y, z\right)\right]\right\rangle \mathrm{d} x_{1} \mathrm{~d} y_{1} \mathrm{~d} x_{2} \mathrm{~d} y_{2}
\end{aligned}
$$

where $z$ refers to the propagation distance in the atmosphere, $k=2 \pi / \lambda$ is the wave number, $\psi\left(x_{1}, y_{1}, x, y\right)$ is the solution to the Rytov method that represents the random part of the complex phase. The angle bracket indicates the ensemble average over the medium statistics covering the log-amplitude and phase fluctuations due to the turbulent atmosphere. The asterisk means the complex conjugation. $A, B, C, D$ denote the elements of the transfer matrix of the optical system. Moreover, there is no inherent aperture between the source and the output planes. Therefore, $A, B, C, D$ are all real-valued.

The phase term $\left\langle\exp \left[\psi\left(x_{1}, y_{1}, x, y\right)+\psi^{*}\left(x_{2}, y_{2}, x, y\right)\right]\right\rangle$ can be written as [25]

$$
\begin{aligned}
& \left\langle\exp \left[\psi\left(x_{1}, y_{1}, x, y\right)+\psi^{*}\left(x_{2}, y_{2}, x, y\right)\right]\right\rangle \\
& \quad=\exp \left\{-\frac{\pi^{2} k^{2} T z}{3}\left[\left(x_{1}-x_{2}\right)^{2}+\left(y_{1}-y_{2}\right)^{2}\right]\right\}
\end{aligned}
$$

where $T$ is a turbulence parameter defined by [25]

$$
T=\frac{A(\alpha)}{2(\alpha-2)} C_{n}^{2}\left[\kappa_{m}^{2-\alpha} \beta(\alpha) \exp \left(\kappa_{0}^{2}-\kappa_{m}^{2}\right) \Gamma_{l}\left(2-\alpha / 2, \kappa_{0}^{2}-\kappa_{m}^{2}\right)-4 \kappa_{0}^{4-\alpha}\right]
$$

where $A(\alpha)$ and $\beta(\alpha)$ are functions of $\alpha(3<\alpha<4)$ and defined by:

$$
A(\alpha)=\frac{\Gamma(\alpha-1) \cos (\alpha \pi / 2)}{4 \pi^{2}}
$$




$$
\beta(\alpha)=2 \kappa_{0}^{2}-2 \kappa_{m}^{2}+\alpha \kappa_{m}^{2}
$$

while

$$
\begin{aligned}
& \kappa_{0}=2 \pi / L_{0} \\
& \kappa_{m}=\frac{1}{l_{0}}\left[\frac{2 \pi}{3} \Gamma(5-\alpha / 2) A(\alpha)\right]^{1 /(\alpha-5)}
\end{aligned}
$$

Here, $l_{0}$ and $L_{0}$ represent the internal and external scales of turbulence, respectively, $\Gamma(\cdot)$ denotes the gamma function, and $\Gamma_{l}(\cdot)$ refers to the incomplete gamma function, $C_{n}^{2}$ is the generalized refractive index structure parameter and describes the turbulence level, with a unit of $\mathrm{m}^{3-\alpha}$. The parameter $\alpha$ represents the power law exponent. Equation (5) is applicable for the description of both Kolmogorov $(\alpha=11 / 3)$ and non-Kolmogorov $(\alpha \neq 11 / 3)$ turbulences.

Inserting Eqs. (1), (2) and (4) into Eq. (3), the average intensity distribution of the partially coherent ChGB through an $A B C D$ optical system in turbulent atmosphere can be obtained by calculating the following integral:

$$
\begin{aligned}
& \langle I(x, y, z)\rangle=\frac{1}{\lambda^{2} B^{2}} \iiint \int W_{t}\left(x_{1}, x_{2}, y_{1}, y_{2}\right) \\
& \times \exp \left\{-\frac{i k}{2 B}\left[A\left(x_{1}^{2}-x_{2}^{2}+y_{1}^{2}-y_{2}^{2}\right)-2 x\left(x_{1}-x_{2}\right)-2 y\left(y_{1}-y_{2}\right)+D x^{2}\right]\right\} \mathrm{d} x_{1} \mathrm{~d} y_{1} \mathrm{~d} x_{2} \mathrm{~d} y_{2}
\end{aligned}
$$

where

$$
W_{t}\left(x_{1}, x_{2}, y_{1}, y_{2}\right)=W_{0}\left(x_{1}, x_{2}, y_{1}, y_{2}\right) \exp \left\{-\frac{\pi^{2} k^{2} T z}{3}\left[\left(x_{1}-x_{2}\right)^{2}+\left(y_{1}-y_{2}\right)^{2}\right]\right\}
$$

To indicate the spatial vector points in a transverse plane, we have used $\boldsymbol{\rho} \equiv(u, v)$ and $\mathbf{r} \equiv(x, y)$ to indicate the spatial vector points in a transverse plane. To indicate discrete coordinates, we set $x_{j_{1}}=j_{1} \Delta_{1}, y_{k_{1}}=k_{1} \Delta_{1}, u_{m}=m \Delta_{1}$ and $v_{n}=n \Delta_{2}$, where the grid (sampling) separations in the input and output planes are $\Delta_{1}$ and $\Delta_{2}$, respectively. The discrete form of Eq. (6) is as follows [21]:

$$
\left\langle I\left(u_{m}, v_{n}, z\right)\right\rangle=[\mathbf{I}]_{m n}=\sum_{j_{1}}^{N_{1}} \sum_{j_{2}}^{N_{1}} \sum_{k_{1}}^{N_{1}} \sum_{k_{2}}^{N_{1}}\left[\mathbf{H}_{y}^{\mathrm{T}}\right]_{n k_{1}}\left[\mathbf{H}_{x}^{\mathrm{T}}\right]_{m j_{1}}\left[W_{t}\right]_{j_{1} j_{2} k_{1} k_{2}}\left[\mathbf{H}_{x}\right]_{j_{2} m}\left[\mathbf{H}_{x}\right]_{k_{2} n}
$$

where the average intensity $\mathbf{I} \equiv\left\{I\left(u_{m}, v_{n}\right)\right\}$ with $m, n=1 \ldots N_{2}$ is a matrix of $N_{2} \times N_{2}$. $\mathbf{H}_{x}=\left\{H_{x}\left(x_{j}, u_{m}\right)\right\}$ and $\mathbf{H}_{y}=\left\{H_{y}\left(y_{k}, v_{n}\right)\right\}$ with $j, k=1 \ldots N_{1}$ represent the impulse response functions of a free propagation system in $x$ and $y$ directions, respectively, and 
both are $N_{1} \times N_{2}$ matrices. $N_{1}$ and $N_{2}$ denote integers, representing the numbers of sampling points in the input and output planes, respectively. $T$ indicates the matrix transpose and complex conjugate.

Within the paraxial approximation, i.e., $\Delta_{1} \ll \sqrt{\lambda z}$, the response matrix through an $A B C D$ system is as follows:

$$
\begin{aligned}
{\left[\mathbf{H}_{x}\right]_{j_{m}} } & \equiv H_{x}\left(x_{j}, u_{m}\right) \\
& =\Delta_{1} \frac{\exp (i \pi B / \lambda)}{\sqrt{i \lambda B}} \operatorname{sinc}\left[\frac{\left(u_{m}-A x_{j}\right) \Delta_{1}}{\lambda B}\right] \exp \left[i \frac{\pi}{\lambda B}\left(A x_{j}^{2}-2 x_{j} u_{m}+D u_{m}^{2}\right)\right]
\end{aligned}
$$

$\mathbf{H}_{y}$ can be represented in the same way. $\mathbf{W}_{t} \equiv\left\{W_{t}\left(x_{j_{1}}, x_{j_{2}}, y_{k_{1}}, y_{k_{2}}\right)\right\}$ with $j_{1,2}, k_{1,2}$ $=1 \ldots N_{1}$ signifies the discrete form of the input CSD function, which is a tensor (multidimensional array) of $N_{1} \times N_{1} \times N_{1} \times N_{1}$. The discrete form of Eq. (7) is as follows:

$$
\left[W_{t}\right]_{j_{1} j_{2} k_{1} k_{2}} \equiv\left[\mathbf{W}_{0}\right]_{j_{1} j_{2} k_{1} k_{2}} \exp \left\{-\frac{\pi^{2} k^{2} T z}{3}\left[\left(x_{j_{1}}^{2}-x_{j_{2}}^{2}\right)+\left(y_{k_{1}}-y_{k_{2}}\right)^{2}\right]\right\}
$$

Because the CSD function of the optical source is $x-y$ separable, Eq. (7) can be separated, i.e., where

$$
W_{t x}\left(x_{1}, x_{2}\right)=W_{0}\left(x_{1}, x_{2}\right) \exp \left\{-\frac{\pi^{2} k^{2} T z}{3}\left(x_{1}-x_{2}\right)^{2}\right\}
$$

And $W_{t y}\left(x_{1}, x_{2}\right)$ can be obtained in a similar way. Equation (8) can be simplified into a matrix form

$$
[I]_{m n}=\left[\mathbf{H}_{x}^{\mathrm{T}} \times \mathbf{W}_{t x} \times \mathbf{H}_{x}\right]_{m m} \cdot\left[\mathbf{H}_{y}^{\mathrm{T}} \times \mathbf{W}_{t y} \times \mathbf{H}_{y}\right]_{n n}
$$

where $\mathbf{W}_{t x}$ and $\mathbf{W}_{t y}$ are $N_{1} \times N_{1}$ matrices: $W_{t x} \equiv\left\{W_{t x}\left(x_{j_{1}}, x_{j_{2}}\right)\right\}, W_{t y} \equiv\left\{W_{t y}\left(y_{k_{1}}, y_{k_{2}}\right)\right\}$.

Through Eq. (12), the evolution of the average intensity of partially coherent ChGB through an $A B C D$ optical system in non-Kolmogorov turbulence can be quantitatively analyzed.

\section{Simulation result and analysis}

In this section, we simulate the propagation of a partially coherent ChGB transmitting through an $A B C D$ optical system in non-Kolmogorov turbulence. The main results of the ETA can be summarized as Eq. (12), which provides the averaged intensity for a partially coherent $\mathrm{ChGB}$ in turbulence. As a numerical example, a partially coherent $\mathrm{ChGB}$ is focused by a lens, propagates through atmospheric turbulence, and is eventually terminated at the observation plane, which is shown in Fig. 1. 


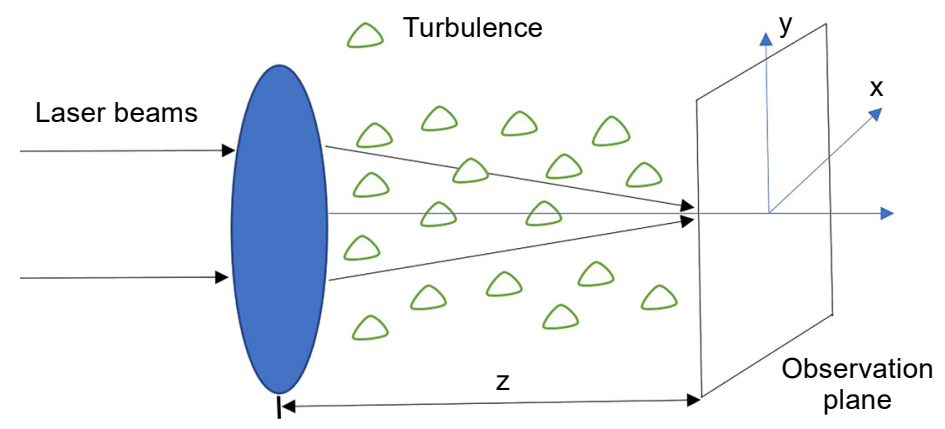

Fig. 1. Schematic diagram of the single lens system in turbulent atmosphere.

The dimension of the thin lens is assumed to be larger than the corresponding beam diameter. Therefore, the diffraction due to the lens is neglected. The distance between the lens and observation plane is $z$. The corresponding transfer matrix between the source plane and the observed plane in the focusing system is [26]

$$
\left(\begin{array}{ll}
A & B \\
C & D
\end{array}\right)=\left(\begin{array}{ll}
1 & z \\
0 & 1
\end{array}\right)\left(\begin{array}{ll}
1 & 0 \\
-1 / f & 1
\end{array}\right)=\left(\begin{array}{ll}
1-z / f & z \\
-1 / f & 1
\end{array}\right)
$$

Substituting Eq. (13) into Eq. (12), we can calculate the propagation properties of a partially coherent ChGB through an $A B C D$ optical system in non-Kolmogorov turbulence numerically. The parameters that do not change in the simulation are selected as $\alpha=3.5, A(\alpha)=0.033, L_{0}=10 \mathrm{~m}, l_{0}=0.001 \mathrm{~m}, \lambda=632.8 \mathrm{~nm}, w_{0}=0.06 \mathrm{~m}$ and $f=50 \mathrm{~m}$ in all figures.

Firstly, the effects of the spatial correlation length of the laser source $\sigma_{0}$ and the propagation distance $z$ on the average intensity distribution of a partially coherent ChGB through an $A B C D$ optical system in non-Kolmogorov turbulence are investigated.

Figure 2 shows calculated results for the average intensity distribution with $\Omega_{0}=$ $=30 \mathrm{~m}^{-1}$ and $C_{n}^{2}=1 \times 10^{-14} \mathrm{~m}^{-2 / 3}$. The curves of black, red, and green correspond to $\sigma_{0}=0.04 \mathrm{~m}, 0.02 \mathrm{~m}$, and $0.01 \mathrm{~m}$, respectively. Subfigure (a), (b), and (c) correspond to $z=1000 \mathrm{~m}, 3000 \mathrm{~m}$, and $5000 \mathrm{~m}$, respectively.

One finds from Fig. 2a that the average intensity distributions of the curves of black, red, and green are very close and all have two peaks when the propagation distance is small $(z=1000 \mathrm{~m})$. With increasing the propagation distance, the average intensity distribution gradually evolves into a Gaussian-like distribution, the width of the beam is gradually expanding and the difference between the three curves increases. This phenomenon could be explained by the fact that the average intensity distribution of the beam is almost independent of the spatial coherence length when the propagation distance is very small. Moreover, it is clear that the peak value of average intensity of 

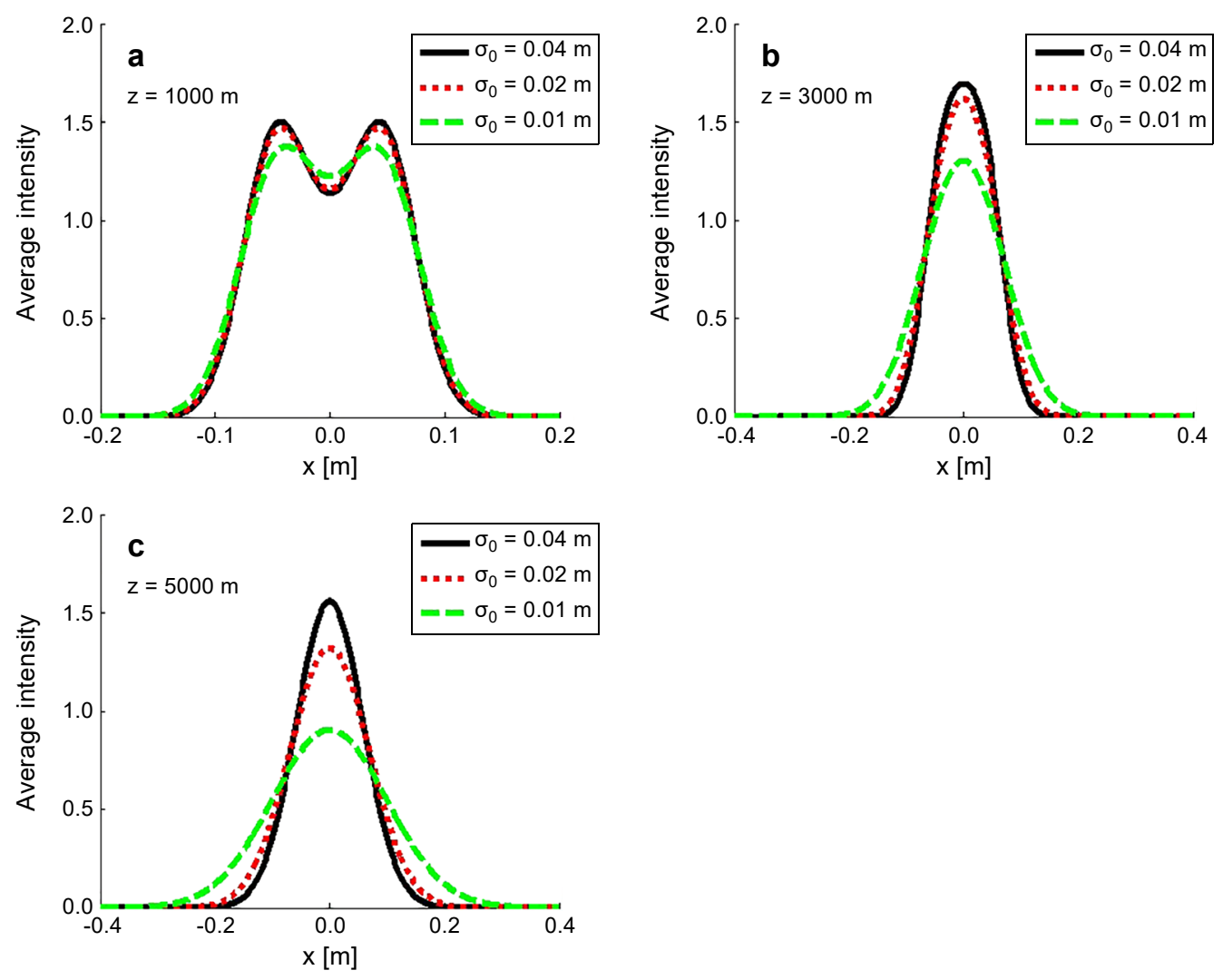

Fig. 2. Average intensity distribution in the $x$ direction of a partially coherent ChGB with different values of spatial correlation length through an $A B C D$ optical system at different propagation distances in turbulent atmosphere at $\Omega_{0}=30 \mathrm{~m}^{-1}$. (a) $z=1000 \mathrm{~m}$, (b) $z=3000 \mathrm{~m}$, and (c) $z=5000 \mathrm{~m}$.

the curve with larger $\sigma_{0}$ is always higher than that of the curve with smaller $\sigma_{0}$ when the propagation distance is long enough. That is to say, the propagation of a partially coherent $\mathrm{ChGB}$ with a larger value of $\sigma_{0}$ is less affected by distance.

Then Fig. 3 illustrates the average intensity distributions when Ch-parameter $\Omega_{0}$ changes from 30 to $10 \mathrm{~m}^{-1}$, and the rest of parameters are same as those in Fig. 2. It is found that the average intensity always maintains a Gaussian-like distribution, which shows $\Omega_{0}$ affects the initial intensity distribution of a partially coherent ChGB. Meanwhile, as the propagation distance increases, the peak value of the average intensity decreases gradually and the width of the beam expands gradually. Particularly, comparing Fig. 2a and Fig. 3a $(z=1000 \mathrm{~m})$, it can be found that as the $\Omega_{0}$ decreases, the peak values of the three curves are all significantly reduced. Same for $z=3000 \mathrm{~m}$ and $z=5000 \mathrm{~m}$, which means the $\Omega_{0}$ has an obvious effect on the average intensity distribution of the ChGB. 

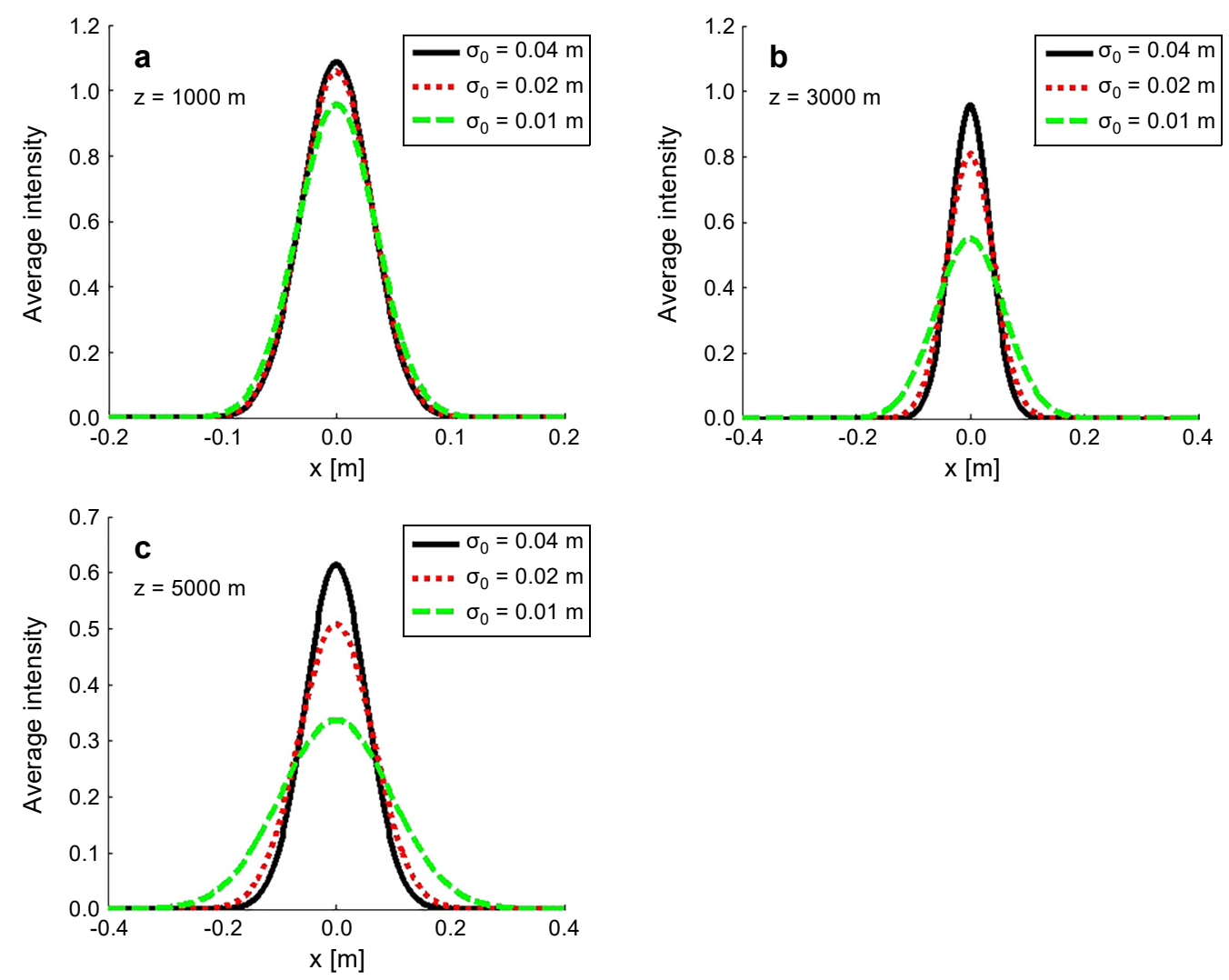

Fig. 3. Average intensity distribution in the $x$ direction of a partially coherent ChGB with different values of spatial correlation length through an $A B C D$ optical system at different propagation distances in turbulent atmosphere at $\Omega_{0}=10 \mathrm{~m}^{-1}$. (a) $z=1000 \mathrm{~m},(\mathbf{b}) z=3000 \mathrm{~m}$, and (c) $z=5000 \mathrm{~m}$.

To further investigate the effect of $\Omega_{0}$ on the average intensity distribution of the beam, Fig. 4 shows the average intensity distribution of a partially coherent ChGB of different values of Ch-parameters $\Omega_{0}$ in turbulent atmosphere with $\sigma_{0}=0.04 \mathrm{~m}$, $z=5000 \mathrm{~m}$, and $C_{n}^{2}=1 \times 10^{-14} \mathrm{~m}^{-2 / 3}$. It is obvious that the peak value of the average intensity increases with the increase of $\Omega_{0}$, that is to say, the beam with larger Ch-parameter $\Omega_{0}$ is less affected by the turbulent atmosphere.

Figure 5 illustrates the average intensity of partially coherent $\mathrm{ChGB}$ with different values of the structure constant of turbulence $C_{n}^{2}$ through an $A B C D$ optical system in non-Kolmogorov turbulence with $\Omega_{0}=10 \mathrm{~m}^{-1}$ and $z=3000 \mathrm{~m}$. The curves of black, and red correspond to $C_{n}^{2}=1 \times 10^{-14} \mathrm{~m}^{-2 / 3}$, and $C_{n}^{2}=1 \times 10^{-16} \mathrm{~m}^{-2 / 3}$, respectively. Subfigure (a), (b), and (c) correspond to $\sigma_{0}=0.04 \mathrm{~m}, 0.02 \mathrm{~m}$ and $0.01 \mathrm{~m}$, respectively. From Fig. 5, it can be seen that with the decreasing of turbulence structure parameter $C_{n}^{2}$ (i.e., turbulence intensity weakening), the peak value of the average intensity decreases 


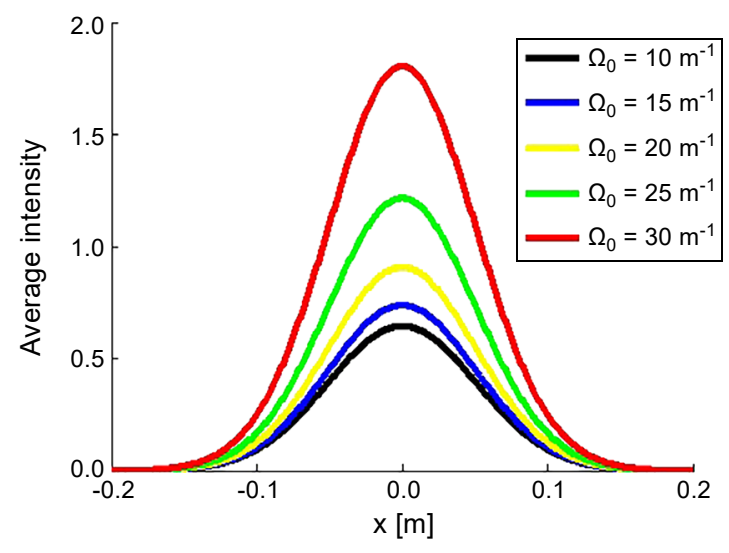

Fig. 4. Average intensity distribution in the $x$ direction of a partially coherent ChGB with different values of Ch-parameters through an $A B C D$ optical system in turbulent atmosphere.
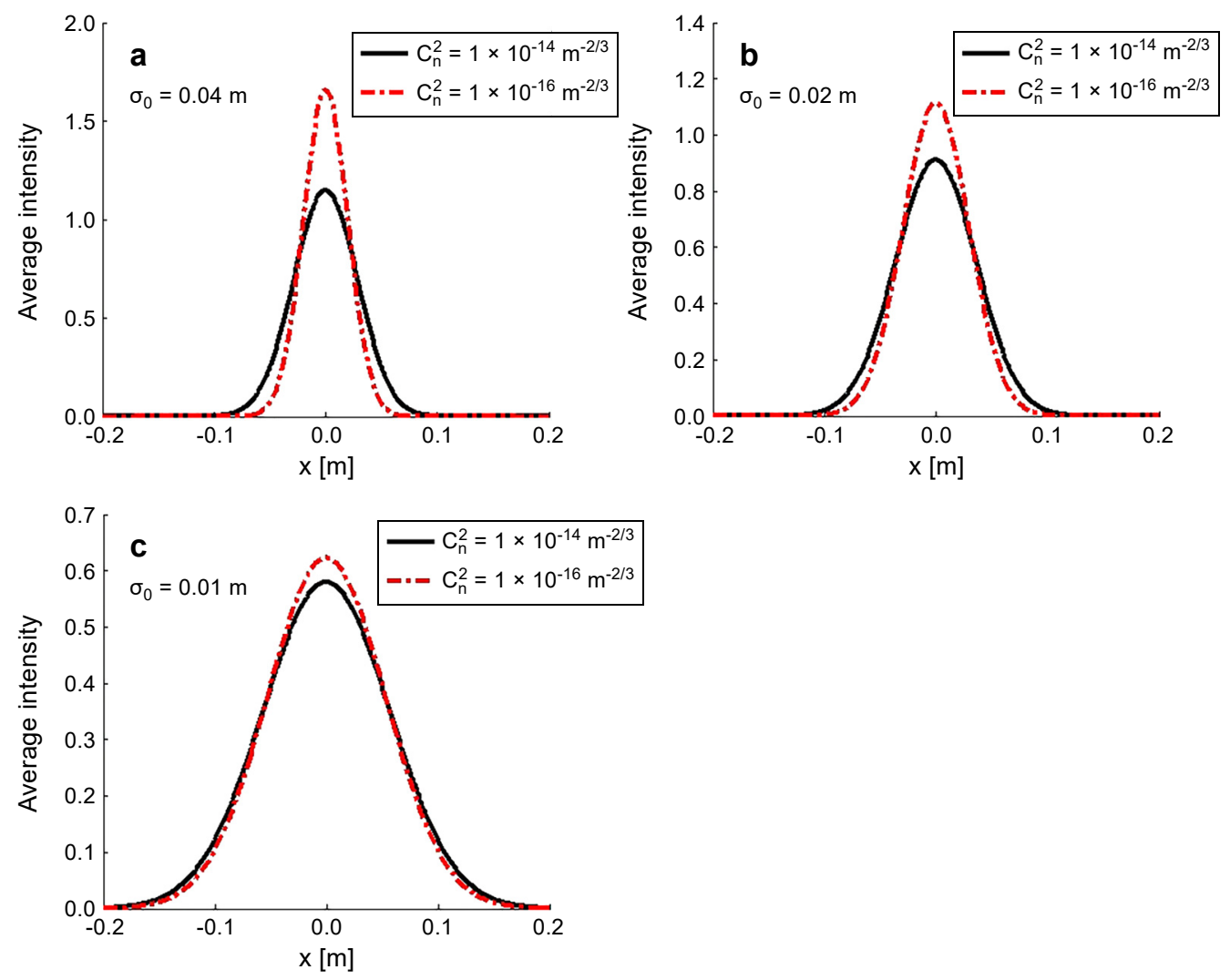

Fig. 5. Average intensity distribution in the $x$ direction of a partially coherent ChGB with different values of spatial correlation length through an $A B C D$ optical system at different turbulence levels. 
obviously. From all three figures (Figs. $5 \mathbf{a}-5 \mathbf{c}$ ), it can be found that the peak value of ChGB with a smaller spatial coherence length $\sigma_{0}$ is lower than that of ChGB with a larger $\sigma_{0}$. However, when $\sigma_{0}=0.01 \mathrm{~m}$, the average intensity distributions of a partially coherent ChGB with $C_{n}^{2}=1 \times 10^{-14} \mathrm{~m}^{-2 / 3}$ and $C_{n}^{2}=1 \times 10^{-16} \mathrm{~m}^{-2 / 3}$ are very close. This phenomenon could be explained by the fact that the turbulent structure constant $C_{n}^{2}$ has little effect on the average intensity distribution of the beam when the spatial coherence length $\sigma_{0}$ is small. Moreover, comparing Figs. $5 \mathbf{a}-5 \mathbf{c}$, it is clear the difference between the two curves at different turbulence levels increases with increasing $\sigma_{0}$, which means the average intensity distribution of the beam is more affected by the structure constant of turbulence (i.e., turbulence level) when the spatial coherence length is larger.

\section{Conclusion}

In conclusion, the propagation of a partially coherent ChGB through $A B C D$ optical system in non-Kolmogorov turbulence is simulated by the effective tensor approach (ETA). Analytical expressions for the cross-spectral density (CSD) and the average intensity are derived. As a numerical example, the properties of the average intensity of a partially coherent ChGB through the optical system of a thin lens in non-Kolmogorov turbulence are demonstrated. The effects of the spatial coherence length $\sigma_{0}$, the Ch-parameter $\Omega_{0}$, the propagation distance $z$ and the structure constant of turbulence $C_{n}^{2}$ on beam propagation are mainly discussed. We have found that the $\sigma_{0}$ has less influence on the average intensity distribution of the beam when the propagation distance is small. Furthermore, the propagation of a partially coherent ChGB with a larger value of $\sigma_{0}$ is less affected by distance when the propagation distance is long enough. It is also found that with the increase of $\Omega_{0}$, the propagation is less affected by turbulent atmosphere. And the average intensity distribution of the beam with larger $\sigma_{0}$ is more affected by the $C_{n}^{2}$ (i.e., turbulence level). By selecting appropriate $\mathrm{Ch}$-parameter and spatial coherence length, the partially coherent ChGB can be better transmitted in the non-Kolmogorov turbulence.

Acknowledgements - This work is supported by the Commonwealth Project from Science Technology Department of Zhejiang Province (No. 2017C33005).

\section{References}

[1] LuKIN I.P., Coherence of Bessel-Gaussian beams propagating in a turbulent atmosphere, Atmospheric and Oceanic Optics 31(1), 2018, pp. 49-59, DOI: 10.1134/S1024856018010098.

[2] Yuan Y., Lei T., Li Z., Li Y., Gao S., Xie Z., Yuan X., Beam wander relieved orbital angular momentum communication in turbulent atmosphere using Bessel beams, Scientific Reports 7, 2017, article 42276, DOI: 10.1038/srep42276.

[3] Avetisyan H., Monken C.H., Mode analysis of higher-order transverse-mode correlation beams in a turbulent atmosphere, Optics Letters 42(1), 2017, pp. 101-104, DOI: 10.1364/OL.42.000101.

[4] SaAd F., El Halba E.M., Belafhal A., A theoretical study of the on-axis average intensity of generalized spiraling Bessel beams in a turbulent atmosphere, Optical and Quantum Electronics 49(3), 2017, article 94, DOI: 10.1007/s11082-017-0936-4. 
[5] Zilberman A., Golbraikh E., Kopeika N.S., Virtser A., Kupershmidt I., Shtemler Y., Lidar study of aerosol turbulence characteristics in the troposphere: Kolmogorov and non-Kolmogorov turbulence, Atmospheric Research 88(1), 2008, pp. 66-77, DOI: 10.1016/j.atmosres.2007.10.003.

[6] Zilberman A., Golbraikh E., Kopeika N.S., Propagation of electromagnetic waves in Kolmogorov and non-Kolmogorov atmospheric turbulence: three-layer altitude model, Applied Optics 47(34), 2008, pp. 6385-6391, DOI: 10.1364/AO.47.006385.

[7] Rao C., JiAng W., Ling N., Spatial and temporal characterization of phase fluctuations in non -Kolmogorov atmospheric turbulence, Journal of Modern Optics 47(6), 2000, pp. 1111-1126, DOI: $10.1080 / 09500340008233408$.

[8] Beland R.R., Some aspects of propagation through weak isotropic non-Kolmogorov turbulence, Proceedings of SPIE 2375, 1995, pp. 6-16, DOI: 10.1117/12.206979.

[9] Toselli I., Andrews L.C., Phillips R.L., Ferrero V., Free space optical system performance for laser beam propagation through non Kolmogorov turbulence for uplink and downlink paths, Proceedings of SPIE 6708, 2007, article 670803, DOI: 10.1117/12.732595.

[10] Toselli I., Andrews L.C., Phillips R.L., Ferrero V., Free-space optical system performance for laser beam propagation through non-Kolmogorov turbulence, Optical Engineering 47(2), 2008, article 026003, DOI: $10.1117 / 1.2870113$.

[11] Wu G., Guo H., Yu S., Luo B., Spreading and direction of Gaussian-Schell model beam through a non-Kolmogorov turbulence, Optics Letters 35(5), 2010, pp. 715-717, DOI: 10.1364/OL.35.000715.

[12] Shchepakina E., Korotкova O., Second-order statistics of stochastic electromagnetic beams propagating through non-Kolmogorov turbulence, Optics Express 18(10), 2010, pp. 10650-10658, DOI: 10.1364/OE.18.010650.

[13] Toselli I., Wang F., KorotKova O., LIDAR systems operating in a non-Kolmogorov turbulent atmosphere, Waves in Random and Complex Media 29(4), 2019, pp. 743-758, DOI: 10.1080/17455030. 2018.1470356.

[14] Korotkova O., Cai Y., Watson E., Stochastic electromagnetic beams for LIDAR systems operating through turbulent atmosphere, Applied Physics B 94(4), 2009, pp. 681-690, DOI: $\underline{10.1007 / \mathrm{s} 00340}$ -009-3404-4.

[15] YuAn Y., YANG Y., Propagation of anomalous vortex beams through an annular apertured paraxial ABCD optical system, Optical and Quantum Electronics 47(7), 2015, pp. 2289-2297, DOI: $10.1007 /$ s11082-014-0105-y.

[16] Casperson L.W., Tovar A.A., Hermite-sinusoidal-Gaussian beams in complex optical systems, Journal of the Optical Society of America A 15(4), 1998, pp. 954-961, DOI: 10.1364/JOSAA.15.000954.

[17] Gao X., Wang Q., Zhan Q., Yun M., Guo H., Zhuang S., Focal patterns of higher order hyperbolic -cosine-Gaussian beam with one optical vortex, Optical and Quantum Electronics 42(6-7), 2011, pp. 367-380, DOI: 10.1007/s11082-011-9468-5.

[18] Li J., Zhuang S., XIE Y., Huang C., Gradient force pattern of truncated hyperbolic-cosine-Gaussian beam through phase plate focusing system, Optical Engineering 46(12), 2007, article 124201, DOI: $10.1117 / 1.2819345$.

[19] Li J., Gao X., Li C., XIE Y., The effect of concentric zones phase plate on focal depth of hyperbolic -cosine-Gaussian beam, Laser Journal 29(1), 2008, pp. 16-18.

[20] Jun C., Zhang E., Peng X., Cai Y., Efficient tensor approach for simulating paraxial propagation of arbitrary partially coherent beams, Optics Express 25(20), 2017, pp. 24780-24789, DOI: 10.1364/OE.25.024780.

[21] CHEN J., Dong W., Efficient tensor approach for propagation of beams of arbitrary shape and coherence through atmospheric turbulence, Optics and Lasers in Engineering 108, 2018, pp. 36-40, DOI: $10.1016 /$ j.optlaseng.2018.04.015.

[22] GBur G., Partially coherent beam propagation in atmospheric turbulence, Journal of the Optical Society of America A 31(9), 2014, pp. 2038-2045, DOI: 10.1364/JOSAA.31.002038.

[23] Schmidt J.D., Numerical Simulation of Optical Wave Propagation with Examples in MATLAB, SPIE, 2010, DOI: $10.1117 / 3.866274$. 
[24] CHU X., The relay propagation of partially coherent cosh-Gaussian-Schell beams in turbulent atmosphere, Applied Physics B 98(2-3), 2010, pp. 573-579, DOI: 10.1007/s00340-009-3769-4.

[25] Zноu G., CHu X., Propagation of a partially coherent cosine-Gaussian beam through an ABCD optical system in turbulent atmosphere, Optics Express 17(13), 2009, pp. 10529-10534, DOI: 10.1364/ OE.17.010529.

[26] Wang S., Zhao D., Matrix Optics, CHEP-Springer, 2000.

Received December 20, 2019

in revised form February 25, 2020 